\title{
Exponential Stabilization of a Swelling Porous-Elastic System with Microtemperature Effect and Distributed Delay
}

\author{
Abdelbaki Choucha, ${ }^{1}$ Salah Mahmoud Boulaaras $\mathbb{D}^{2,3}$ Djamel Ouchenane, ${ }^{4}$ \\ Bahri Belkacem Cherif $\mathbb{D}^{\mathrm{D}},{ }^{2,5}$ Muajebah Hidan ${ }^{\mathrm{D}}{ }^{6}{ }^{6}$ and Mohamed Abdalla ${ }^{6,7}$ \\ ${ }^{1}$ Laboratory of Operator Theory and PDEs: Foundations and Applications, Department of Mathematics, Faculty of Exact Sciences, \\ University of El Oued, Box 789, El Oued 39000, Algeria \\ ${ }^{2}$ Department of Mathematics, College of Sciences and Arts, ArRas, Qassim University, Saudi Arabia \\ ${ }^{3}$ Laboratory of Fundamental and Applied Mathematics of Oran (LMFAO), University of Oran 1, Ahmed Benbella, Algeria \\ ${ }^{4}$ Laboratory of Pure and Applied Mathematics, Amar Teledji Laghouat University, Algeria \\ ${ }^{5}$ Preparatory Institute for Engineering Studies in Sfax, Tunisia \\ ${ }^{6}$ Mathematics Department, College of Science, King Khalid University, Saudi Arabia \\ ${ }^{7}$ Mathematics Department, Faculty of Science, South Valley University, Qena 83523, Egypt
}

Correspondence should be addressed to Bahri Belkacem Cherif; bahi1968@yahoo.com

Received 4 February 2021; Accepted 8 May 2021; Published 19 May 2021

Academic Editor: Shengda Zeng

Copyright (c) 2021 Abdelbaki Choucha et al. This is an open access article distributed under the Creative Commons Attribution License, which permits unrestricted use, distribution, and reproduction in any medium, provided the original work is properly cited.

The swelling porous thermoelastic system with the presence of temperatures, microtemperature effect, and distributed delay terms is considered. We will establish the well posedness of the system, and we prove the exponential stability result.

\section{Introduction and Preliminaries}

Eringen was the first to present a theory in which a mixture of viscous liquid and solids mixed with gas [1]. Then, after studying this heat-resistant mixture, you get to the field equations [2].

Expansive (swelling) soils have also been classified under porous media theory which studies this type of problem. This is why this field is considered fertile for study, as there are many studies to reduce the damage caused by swelling soil, especially in civil engineering and architecture, for more depth (see [3-8]).

The basic field equations of the linear theory of swelling porous elastic soils were presented by

$$
\begin{aligned}
& \rho_{u} u_{t t}=P_{1 x}+G_{1}+H_{1}, \\
& \rho_{\phi} \phi_{t t}=P_{2 x}+G_{2}+H_{2},
\end{aligned}
$$

where $u, \phi$ are the displacement of the fluid and the elastic solid material and $\rho_{u}, \rho_{\phi}>0$ are the densities of each constituent. And $\left(P_{1}, G_{1}, H_{1}\right)$ are the partial tension, internal body forces, and eternal forces acting on the displacement, respectively, similarly $\left(P_{2}, G_{2}, H_{2}\right)$, but acting on the elastic solid. In addition, the constitutive equations of partial tensions are given by

$$
\left(\begin{array}{c}
P_{1} \\
P_{2}
\end{array}\right)=\underbrace{\left(\begin{array}{l}
a_{1}, a_{2} \\
a_{2}, a_{3}
\end{array}\right)}_{A} \cdot\left(\begin{array}{l}
u_{x} \\
\phi_{x}
\end{array}\right),
$$

where $a_{1}, a_{3}>0$ and $a_{2} \neq 0$ are real numbers. $A$ is matrix positive definite with $a_{1} a_{3}>a_{2}^{2}$.

Quintanilla [8] investigated (2) by taking

$$
G_{1}=G_{2}=\xi\left(u_{t}-\phi_{t}\right), H_{1}=a_{3} u_{x x t}, H_{2}=0 \text {, }
$$

where $\xi>0$; they obtained the stability is exponentially. 
Similarly, in [9], the authors are considered (2) with a different conditions

$$
G_{1}=G_{2}=0, \quad H_{1}=-\rho_{u} \gamma(x) u_{t}, \quad H_{2}=0,
$$

where $\gamma(x)$ is an internal viscous damping function with positive mean. By the spectral method, they obtained the exponential stability result. For more details, see [8-15].

Time delays are very important in most natural phenomena and industrial devices, where the time lag is a source of instability, and it is a problem worthy of attention.

Also, there are many works that have studied this type of problems, of which $[11,15-24]$.

The basic evolution equations for one-dimensional theories of swelling porous materials with temperature and microtemperature [25-28] are given by

$$
\begin{aligned}
\rho_{u} u_{t t} & =T_{x}, \\
\rho_{\varphi} \phi_{t t} & =H_{x}+G, \\
\rho \eta_{t} & =q_{x}, \\
\rho E_{t} & =P_{x}^{*}+q-Q .
\end{aligned}
$$

Here, $T, H, G, q, \eta, P^{*}, Q$, and $E$ represent the stress, the equilibrated stress, the equilibrated body force, the heat flux vector, the entropy, the first heat flux moment, the mean heat flux, and the first moment of energy. The constitutive equations are

$$
\begin{aligned}
& T=P_{1}+G_{1}+H_{1} P^{*}=-k_{2} w_{x}, \\
& H=P_{2}+P_{3} \rho \eta=\gamma u_{x}+c_{0} \theta+m \phi, \\
& G=G_{2}+H_{2} Q=-k_{3} w-k_{1} \theta_{x}, \\
& q=\kappa \theta_{x} \rho E=-\alpha w-d \phi_{x},
\end{aligned}
$$

where $w$ is the microtemperature vector, $\rho_{u}, \rho_{\phi}, k_{1}, k_{2}, k_{3}$, $a_{1}, a_{2}, a_{3}, \alpha, \kappa, c_{0}, \mu_{1}>0$. As coupling is considered, $a_{2} \neq 0$ and satisfies

$$
a=a_{3}-\frac{a_{2}^{2}}{a_{1}}>0 .
$$

And the coefficients $\gamma, m, d>0$.

The goal of this work is the thermal effects, so we suppose that the heat capacity $c_{0}>0$, and for more excitement in posing the problem, we suppose that the thermal conductivity is nonexistent $\kappa \neq 0$.

And by introducing the distributed delay term, form a new problem different from previous studies. Under appropriate suppositions, the well posedness of the system is established, and we prove the exponential stability result by the energy method.

We consider in this work:

$$
G_{1}=G_{2}=0, \quad P_{3}=-d w,
$$

$$
\begin{aligned}
& H_{1}=-\gamma \theta \\
& H_{2}=m \theta-\mu_{1} \phi_{t}-\int_{\tau_{1}}^{\tau_{2}}\left|\mu_{2}(\sigma)\right| \phi_{t}(x, t-\sigma) d \sigma .
\end{aligned}
$$

Now, by substituting (13)-(17) into (9), we arrive at the following problem:

$$
\left\{\begin{array}{l}
\rho_{u} u_{t t}-a_{1} u_{x x}-a_{2} \phi_{x x}-\gamma \theta_{x}=0, \\
\rho_{\phi} \phi_{t t}-a_{3} \phi_{x x}-a_{2} u_{x x}-d w_{x}+m \theta+\mu_{1} \phi_{t}+\int_{\tau_{1}}^{\tau_{2}}\left|\mu_{2}(\sigma)\right| \phi_{t}(x, t-\sigma) d \sigma=0, \\
c_{0} \theta_{t}=-\gamma u_{t x}-m \phi_{t}-k_{1} w_{x}, \\
\alpha w_{t}=k_{2} w_{x x}-k_{3} w-k_{1} \theta_{x}-d \phi_{t x},
\end{array}\right.
$$

where

$$
(x, \sigma, t) \in \mathscr{H}=(0,1) \times\left(\tau_{1}, \tau_{2}\right) \times(0, \infty),
$$

under the initial and boundary conditions

$$
\begin{aligned}
u(x, 0) & =u_{0}(x), u_{t}(x, 0)=u_{1}(x), \theta(x, 0)=\theta_{0}(x) \\
\phi(x, 0) & =\phi_{0}(x), \phi_{t}(x, 0)=\phi_{1}(x), w(x, 0)=w_{0}(x), \quad x \in(0,1), \\
\phi_{t}(x,-t) & =f_{0}(x, t), \quad(x, t) \in(0,1) \times\left(0, \tau_{2}\right), \\
u(0, t) & =u(1, t)=\phi(0, t)=\phi(1, t)=0, \\
\theta(0, t) & =\theta(1, t)=w_{x}(0, t)=w_{x}(1, t)=0, \quad t \geq 0 .
\end{aligned}
$$

First, as in [24], we introduce the new variable

$$
\mathscr{Y}(x, \rho, \sigma, t)=\phi_{t}(x, t-\sigma \rho)
$$

Then, we get

$$
\left\{\begin{array}{l}
\sigma \mathscr{Y}_{t}(x, \rho, \sigma, t)+\mathscr{Y}_{\rho}(x, \rho, \sigma, t)=0, \\
\mathscr{y}(x, 0, \sigma, t)=\phi_{t}(x, t) .
\end{array}\right.
$$

Consequently, our problem is written in the form

$$
\left\{\begin{array}{l}
\rho_{u} u_{t t}-a_{1} u_{x x}-a_{2} \phi_{x x}-\gamma \theta_{x}=0, \\
\rho_{\phi} \phi_{t t}-a_{3} \phi_{x x}-a_{2} u_{x x}-d w_{x}+m \theta+\mu_{1} \phi_{t}+\int_{\tau_{1}}^{\tau_{2}}\left|\mu_{2}(\sigma)\right| \mathscr{Y}(x, 1, \sigma, t) d \sigma=0, \\
c_{0} \theta_{t}=-\gamma u_{t x}-m \phi_{t}-k_{1} w_{x}, \\
\alpha w_{t}=k_{2} w_{x x}-k_{3} w-k_{1} \theta_{x}-d \phi_{t x}, \\
\sigma \mathscr{Y}_{t}(x, \rho, \sigma, t)+\mathscr{Y}_{\rho}(x, \rho, \sigma, t)=0 .
\end{array}\right.
$$

where

$$
(x, \rho, \sigma, t) \in(0,1) \times \mathscr{H},
$$


with the initial data

$$
\left\{\begin{array}{l}
u(x, 0)=u_{0}(x), u_{t}(x, 0)=u_{1}(x), \theta(x, 0)=\theta_{0}(x), \\
\phi(x, 0)=\phi_{0}(x), \phi_{t}(x, 0)=\phi_{1}(x), w(x, 0)=w_{0}(x) x \in(0,1), \\
\mathscr{Y}(x, \rho, \sigma, 0)=\mathrm{f}_{0}(x, \rho \sigma),(x, \rho, \sigma) \in(0,1) \times(0,1) \times\left(0, \tau_{2}\right),
\end{array}\right.
$$

and the boundary conditions

$$
\left\{\begin{array}{l}
u(0, t)=u(1, t)=\phi(0, t)=\phi(1, t)=0, \\
\theta(0, t)=\theta(1, t)=w_{x}(0, t)=w_{x}(1, t)=0, \quad t \geq 0 .
\end{array}\right.
$$

Here, the integral represent the distributed delay terms with $\tau_{1}, \tau_{2}>0$ are a time delay; $\mu_{2}$ is an $L^{\infty}$ function satisfying:

(H1) $\mu_{2}:\left[\tau_{1}, \tau_{2}\right] \longrightarrow \mathbb{R}$ is a bounded function satisfying

$$
\int_{\tau_{1}}^{\tau_{2}}\left|\mu_{2}(\sigma)\right| d \sigma<\mu_{1}
$$

Meanwhile, from $(23)_{4}$ and (26), it follows that

$$
\frac{d}{d t} \int_{0}^{1} \omega(x, t) d x+\frac{k_{3}}{\alpha} \int_{0}^{1} \omega(x, t) d x=0 .
$$

So, by solving (28) and using the initial data of $u$, we get

$$
\int_{0}^{1} \omega(x, t) d x=\left(\int_{0}^{1} \omega_{0}(x) d x\right) e^{-(t / \alpha) k_{3}} .
$$

Consequently, if we let

$$
\bar{\omega}(x, t)=\omega(x, t)-\left(\int_{0}^{1} \omega_{0}(x) d x\right) e^{-(t / \alpha) k_{3}},
$$

we get

$$
\int_{0}^{1} \bar{\omega}(x, t) d x=0, \quad \forall t \geq 0
$$

Therefore, the use of Poincare's inequality for $\bar{\omega}$ is justified. In addition, simple substitution shows that $(u, \phi, \theta, \bar{\omega}$, $\mathcal{Y}$ ) satisfies system (23). Henceforth, we work with $\bar{\omega}$ instead of $\omega$ but write $\omega$ for simplicity of notation.

In this paper, we consider $(u, \phi, \theta, w, y)$ to be a solution of system (23)-(26) with the regularity needed to justify the calculations. In Section 2, the well posedness is established, and in Section 3, the exponential stability is proved. In all of the following, we mention that $c>0$.

Remark 1. The coupling that we have proposed in this work with the presence of microtemperatures and distributed delay in problems of swelling in porous elasticity we believe constitutes a new contribution and differs from the previous studies.

\section{Well Posedness}

In this section, we established the well posedness of the system (23)-(26).

First, introducing the vector function

$$
U=\left(u, u_{t}, \phi, \phi_{t}, \theta, w, \mathcal{Y}\right)^{T},
$$

and the variables $v=u_{t}, \varphi=\phi_{t}$, then the system (23) writes as follows:

$$
\left\{\begin{array}{l}
U_{t}=\mathscr{A} U \\
U(0)=U_{0}=\left(u_{0}, u_{1}, \phi_{0}, \phi_{1}, \theta_{0}, w_{0}, f_{0}\right)^{T},
\end{array}\right.
$$

where $\mathscr{A}: \mathscr{D}(\mathscr{A}) \subset \mathscr{H}: \longrightarrow \mathscr{H}$ is the linear operator given by

$$
\mathscr{A} U=\left(\begin{array}{c}
v \\
-\frac{1}{\rho_{u}}\left[a_{1} u_{x x}+a_{2} \phi_{x x}-\gamma \theta_{x}\right] \\
\varphi \\
\frac{1}{\rho_{\phi}}\left[a_{3} \phi_{x x}+a_{2} u_{x x}-d w_{x}+m \theta-\mu_{1} \varphi-\int_{\tau_{1}}^{\tau_{2}}\left|\mu_{2}(\sigma)\right| \mathcal{Y}(x, 1, \sigma, t) d \sigma\right] \\
-\frac{1}{c_{0}}\left[\gamma v_{x}+m \varphi+k_{1} w_{x}\right] \\
\frac{1}{\alpha}\left[k_{2} w_{x x}-k_{3} w-k_{1} \theta_{x}-d \varphi_{x}\right] \\
-\frac{1}{\sigma} \mathscr{Y}_{\rho}
\end{array}\right),
$$

and $\mathscr{H}$ is the energy space given by

$$
\begin{aligned}
\mathscr{H}= & H_{0}^{1}(0,1) \times L^{2}(0,1) \times H_{0}^{1}(0,1) \times L^{2}(0,1) \\
& \times L^{2}(0,1) \times L_{*}^{2}(0,1) \times L^{2}\left((0,1) \times(0,1) \times\left(\tau_{1}, \tau_{2}\right)\right),
\end{aligned}
$$

where

$$
\begin{aligned}
& L_{*}^{2}(0,1)=\left\{\psi \in \frac{L^{2}(0,1)}{\int_{0}^{1} \psi(x) d x}=0\right\}, \\
& H_{*}^{1}(0,1)=H^{1}(0,1) \cap L_{*}^{2}(0,1), \\
& H_{*}^{2}(0,1)=\left\{\psi \in \frac{H^{2}(0,1)}{\psi_{x}(1)}=\psi_{x}(0)=0\right\} .
\end{aligned}
$$

For any,

$$
\begin{aligned}
& U=(u, v, \phi, \varphi, \theta, w, \mathscr{Y})^{T} \in \mathscr{H}, \\
& \widehat{U}=(u \wedge, v \wedge, \phi \wedge, \varphi \wedge, \theta \wedge, w \wedge, \mathscr{Y} \wedge)^{T} \in \mathscr{H},
\end{aligned}
$$

we equip $\mathscr{H}$ with the inner product defined by 


$$
\begin{aligned}
<U, \widehat{U}>_{\mathscr{H}}= & \rho_{u} \int_{0}^{1} v \widehat{v} d x+a_{1} \int_{0}^{1} u_{x} \widehat{u}_{x} d x+\rho_{\phi} \int_{0}^{1} \varphi \widehat{\varphi} d x \\
& +a_{3} \int_{0}^{1} \phi_{x} \widehat{\phi}_{x} d x+c_{0} \int_{0}^{1} \theta \widehat{\theta} d x \\
& +\alpha \int_{0}^{1} w \widehat{w} d x+a_{2} \int_{0}^{1}\left(u_{x} \widehat{\phi}+\widehat{u}_{x} \phi\right) d x \\
& +\int_{0}^{1} \int_{0}^{1} \int_{\tau_{1}}^{\tau_{2}} \sigma\left|\mu_{2}(\sigma)\right| \mathcal{Y} \hat{y} d \sigma d \rho d x .
\end{aligned}
$$

The domain of $\mathscr{A}$ is given by

$$
\mathscr{D}(\mathscr{A})=\left\{\begin{array}{c}
U \in \mathscr{H} \mid u, \phi \in H^{2}(0,1) \cap H_{0}^{1}(0,1), v, \varphi, \theta \in H_{0}^{1}(0,1), \\
w \in H_{*}^{2}(0,1) \cap H_{*}^{1}(0,1), \\
\mathscr{Y}, \mathscr{Y}_{\rho} \in L^{2}\left((0,1) \times(0,1) \times\left(\tau_{1}, \tau_{2}\right)\right), \mathscr{Y}(x, 0, \sigma, t)=\varphi
\end{array}\right\} .
$$

Clearly, $\mathscr{D}(\mathscr{A})$ is dense in $\mathscr{H}$.

Theorem 2. Let $U_{0} \in \mathscr{H}$ and assume that (27) holds. Then, there exists a unique solution $U \in \mathscr{C}\left(\mathbb{R}_{+}, \mathscr{H}\right)$ of problem (33).

Moreover, if $U_{0} \in \mathscr{D}(\mathscr{A})$, then

$$
U \in \mathscr{C}\left(\mathbb{R}_{+}, \mathscr{D}(\mathscr{A})\right) \cap \mathscr{C}^{1}\left(\mathbb{R}_{+}, \mathscr{H}\right) .
$$

Proof. First, we prove that the operator $\mathscr{A}$ is dissipative. For any $U_{0} \in \mathscr{D}(\mathscr{A})$ and by using (38), we have

$$
\begin{aligned}
<\mathscr{A} U, U\rangle_{\mathscr{H}}= & -\mu_{1} \int_{0}^{1} \varphi^{2} d x-\int_{0}^{1} \int_{\tau_{1}}^{\tau_{2}}\left|\mu_{2}(\sigma)\right| \varphi \mathcal{Y}(x, 1, \sigma, t) d \sigma d x \\
& -\int_{0}^{1} \int_{0}^{1} \int_{\tau_{1}}^{\tau_{2}}\left|\mu_{2}(\sigma)\right| \mathscr{Y}_{\rho} \mathcal{Y} d \sigma d \rho d x \\
& -k_{2} \int_{0}^{1} w_{x}^{2} d x-k_{3} \int_{0}^{1} w^{2} d x .
\end{aligned}
$$

For the third term of the RHS of (41), we have

$$
\begin{aligned}
& -\int_{0}^{1} \int_{0}^{1} \int_{\tau_{1}}^{\tau_{2}}\left|\mu_{2}(\sigma)\right| \mathscr{Y}_{\rho} \mathcal{Y} d \sigma d \rho d x \\
& =-\frac{1}{2} \int_{0}^{1} \int_{\tau_{1}}^{\tau_{2}} \int_{0}^{1}\left|\mu_{2}(\sigma)\right| \frac{d}{d \rho} \mathscr{Y}^{2} d \rho d \sigma d x \\
& =-\frac{1}{2} \int_{0}^{1} \int_{\tau_{1}}^{\tau_{2}}\left|\mu_{2}(\sigma)\right| \mathscr{Y}^{2}(x, 1, \sigma, t) d \sigma d x \\
& \quad+\frac{1}{2} \int_{0}^{1} \int_{\tau_{1}}^{\tau_{2}}\left|\mu_{2}(\sigma)\right| \mathscr{Y}^{2}(x, 0, \sigma, t) d \sigma d x
\end{aligned}
$$

By using Young's inequality, we get

$$
\begin{aligned}
-\int_{0}^{1} \int_{\tau_{1}}^{\tau_{2}}\left|\mu_{2}(\sigma)\right| \varphi \mathscr{Y}(x, 1, \sigma, t) d \sigma d x \\
\leq \frac{1}{2}\left(\int_{\tau_{1}}^{\tau_{2}}\left|\mu_{2}(\sigma)\right| d \sigma\right) \int_{0}^{1} \varphi^{2} d x \\
\quad+\frac{1}{2} \int_{0}^{1} \int_{\tau_{1}}^{\tau_{2}}\left|\mu_{2}(\sigma)\right| \mathscr{Y}^{2}(x, 1, \sigma, t) d \sigma d x .
\end{aligned}
$$

Substituting (42), (43) into (41), using $\mathscr{Y}(x, 0, \sigma, t)=$ $\varphi(x, t)$ and (27), we find

$$
<\mathscr{A} U, U>_{\mathscr{H}} \leq-\eta_{0} \int_{0}^{1} \varphi^{2} d x-k_{2} \int_{0}^{1} w_{x}^{2} d x-k_{3} \int_{0}^{1} w^{2} d x \leq 0,
$$

where $\eta_{0}=\left(\mu_{1}-\int_{\tau_{1}}^{\tau_{2}}\left|\mu_{2}(\sigma)\right| d \sigma\right)>0$. Hence, $\mathscr{A}$ is dissipative operator.

Next, we prove $\mathscr{A}$ is maximal operator. It is sufficient to show that $(\lambda I-\mathscr{A})$ is surjective operator.

Indeed, for any $F=\left(f_{1}, f_{2}, f_{3}, f_{4}, f_{5}, f_{6}, f_{7}\right)^{T} \in \mathscr{H}$, we prove that there exists a unique $U=(u, v, \phi, \varphi, \theta, w, z) \epsilon$ $\mathscr{D}(\mathscr{A})$ such that

$$
(\lambda I-\mathscr{A}) U=F
$$

That is,

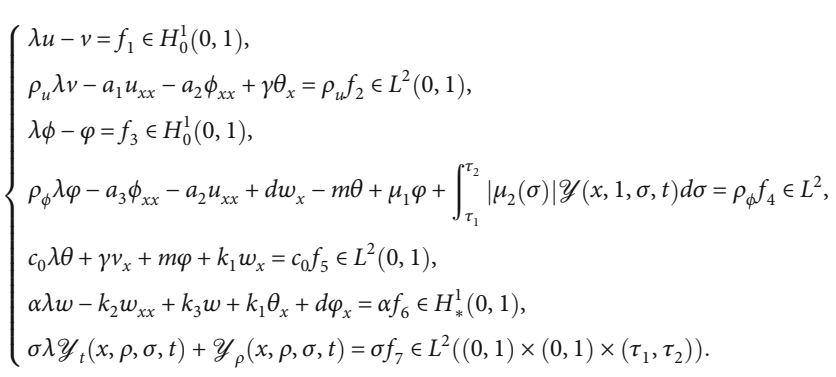

We note that Equation $(46)_{7}$ with $\mathcal{Y}(x, 0, \sigma, t)=\varphi(x, t)$ has a unique solution defined by

$$
\mathcal{Y}(x, \rho, \sigma, t)=e^{-\lambda \rho \sigma} \varphi+\sigma e^{\sigma \rho \lambda} \int_{0}^{\rho} e^{\lambda \sigma \rho} f_{7}(x, \rho, \sigma, t) d \rho
$$

then

$$
\mathcal{Y}(x, 1, \sigma, t)=e^{-\lambda \sigma} \varphi+\sigma e^{\lambda \sigma} \int_{0}^{1} e^{\lambda \sigma \rho} f_{7}(x, \rho, \sigma, t) d \rho,
$$

and we have

$$
v=\lambda u-f_{1}, \quad \varphi=\lambda \phi-f_{3} .
$$


Inserting (48) and (49) in $(46)_{2},(46)_{4},(46)_{5}$, and $(46)_{6}$, we get

$$
\left\{\begin{array}{l}
\rho_{u} \lambda^{2} u-a_{1} u_{x x}-a_{2} \phi_{x x}+\gamma \theta_{x}=h_{1}, \\
\mu_{3} \phi-a_{3} \phi_{x x}-a_{2} u_{x x}+d w_{x}-m \theta=h_{2}, \\
c_{0} \theta+\gamma u_{x}+m \phi+\frac{k_{1}}{\lambda} w_{x}=h_{3}, \\
\frac{\alpha \lambda+k_{3}}{\lambda} w-\frac{k_{2}}{\lambda} w_{x x}+\frac{k_{1}}{\lambda} \theta_{x}+d \phi_{x}=h_{4},
\end{array}\right.
$$

where

$$
\left\{\begin{aligned}
h_{1} & =\rho_{u}\left(\lambda f_{1}+f_{2}\right), \\
h_{2}= & \left(\rho_{\phi} \lambda+\mu_{1}+\int_{\tau_{1}}^{\tau_{2}}\left|\mu_{2}(\sigma)\right| e^{-\sigma \lambda} d \sigma\right) f_{3} \\
& -\int_{\tau_{1}}^{\tau_{2}} \sigma\left|\mu_{2}(\sigma)\right| e^{\sigma \lambda} \int_{0}^{1} e^{\lambda \sigma \rho} f_{7}(x, \rho, \sigma, t) d \rho d \sigma, \\
h_{3}= & \frac{1}{\lambda}\left(\gamma f_{1 x}+m f_{3}+c_{0} f_{5}\right), \\
h_{4}= & \frac{1}{\lambda}\left(\alpha f_{6}+d \lambda f_{3}\right), \\
\mu_{3}= & \rho_{\phi} \lambda^{2}+\mu_{1} \lambda+\lambda \int_{\tau_{1}}^{\tau_{2}}\left|\mu_{2}(\sigma)\right| e^{-\lambda \sigma} d \sigma .
\end{aligned}\right.
$$

We multiply (50) by $\widehat{u}, \widehat{\phi}, \widehat{\theta}, \widehat{w}$, respectively, and integrate their sum over $(0,1)$ to find the following variational formulation:

$$
B((u, \phi, \theta, w),(\widehat{u}, \widehat{\phi}, \widehat{\theta}, \widehat{w}))=\Gamma(\widehat{u}, \widehat{\phi}, \widehat{\theta}, \widehat{w})
$$

where

$$
B:\left(H_{0}^{1}(0,1) \times H_{0}^{1}(0,1) \times L^{2}(0,1) \times H_{*}^{1}(0,1)\right)^{2} \longrightarrow \mathbb{R},
$$

is the bilinear form given by

$$
\begin{aligned}
& B((u, \phi, \theta, w),(\widehat{u}, \widehat{\phi}, \widehat{\theta}, \widehat{w}))=\rho_{u} \lambda^{2} \int_{0}^{1} u \hat{u} d x+a_{1} \int_{0}^{1} u_{x} \widehat{u}_{x} d x \\
& \quad+a_{2} \int_{0}^{1} \phi_{x} \widehat{u}_{x} d x+\gamma \int_{0}^{1} \theta_{x} \widehat{u} d x+\mu_{3} \int_{0}^{1} \phi \widehat{\phi} d x \\
& \quad+a_{3} \int_{0}^{1} \phi_{x} \widehat{\phi}_{x} d x+a_{2} \int_{0}^{1} u_{x} \widehat{\phi}_{x} d x+d \int_{0}^{1} w_{x} \widehat{\phi} d x-m \int_{0}^{1} \theta \widehat{\phi} d x \\
& \quad+c_{0} \int_{0}^{1} \theta \widehat{\theta} d x+\gamma \int_{0}^{1} u_{x} \widehat{\theta} d x+m \int_{0}^{1} \phi \widehat{\theta} d x+\frac{k_{1}}{\lambda} \int_{0}^{1} w_{x} \widehat{\theta} d x \\
& \quad+\frac{\alpha \lambda+k_{3}}{\lambda} \int_{0}^{1} w \widehat{w} d x+\frac{k_{2}}{\lambda} \int_{0}^{1} w_{x} \widehat{w}_{x} d x+\frac{k_{1}}{\lambda} \int_{0}^{1} \theta_{x} \widehat{w} d x+d \int_{0}^{1} \phi_{x} \widehat{w} d x,
\end{aligned}
$$

and

$$
\Gamma:\left(H_{0}^{1}(0,1) \times H_{0}^{1}(0,1) \times L^{2}(0,1) \times H_{*}^{1}(0,1)\right) \longrightarrow \mathbb{R},
$$

is the linear functional defined by

$$
\Gamma(\widehat{u}, \widehat{\phi}, \widehat{\theta}, \widehat{w})=\int_{0}^{1} h_{1} \widehat{u} d x+\int_{0}^{1} h_{2} \widehat{\phi} d x+\int_{0}^{1} h_{3} \widehat{\theta} d x+\int_{0}^{1} h_{4} \widehat{w} d x .
$$

Now, for $V=H_{0}^{1}(0,1) \times H_{0}^{1}(0,1) \times L^{2}(0,1) \times H_{*}^{1}(0,1)$, equipped with the norm,

$$
\begin{aligned}
\|u, \phi, \theta, w\|_{V}^{2}= & \|u\|_{2}^{2}+\left\|u_{x}\right\|_{2}^{2}+\|\phi\|_{2}^{2}+\left\|\phi_{x}\right\|_{2}^{2} \\
& +\|\theta\|_{2}^{2}+\left\|w_{x}\right\|_{2}^{2}+\|w\|_{2}^{2}
\end{aligned}
$$

then we have

$$
\begin{aligned}
& B((u, \phi, \theta, w),(u, \phi, \theta, w))=\rho_{u} \lambda^{2} \int_{0}^{1} u^{2} d x+a_{1} \int_{0}^{1} u_{x}^{2} d x \\
& \quad+\mu_{3} \int_{0}^{1} \phi^{2} d x+a_{3} \int_{0}^{1} \phi_{x}^{2} d x+2 a_{2} \int_{0}^{1} u_{x} \phi_{x} d x \\
& \quad+c_{0} \int_{0}^{1} \theta^{2} d x+\frac{\alpha \lambda+k_{3}}{\lambda} \int_{0}^{1} w^{2} d x+\frac{k_{2}}{\lambda} \int_{0}^{1} w_{x}^{2} d x
\end{aligned}
$$

On the other hand, we can write

$$
\begin{aligned}
a_{1} u_{x}^{2}+2 a_{2} u_{x} \phi_{x}+a_{3} \phi_{x}^{2}= & \frac{1}{2}\left[a_{1}\left(u_{x}+\frac{a_{2}}{a_{3}} \phi_{x}\right)^{2}+a_{3}\left(\phi_{x}+\frac{a_{2}}{a_{1}} u_{x}\right)^{2}\right. \\
& \left.+u_{x}^{2}\left(a_{1}+\frac{a_{2}^{2}}{a_{3}}\right)+\phi_{x}^{2}\left(a_{3}+\frac{a_{2}^{2}}{a_{1}}\right)\right] .
\end{aligned}
$$

Since (14), we deduce

$$
a_{1} u_{x}^{2}+2 a_{2} u_{x} \phi_{x}+a_{3} \phi_{x}^{2}>\frac{1}{2}\left[u_{x}^{2}\left(a_{1}+\frac{a_{2}^{2}}{a_{3}}\right)+\phi_{x}^{2}\left(a_{3}+\frac{\mathrm{a}_{2}^{2}}{a_{1}}\right)\right],
$$

then, for some $M_{0}>0$

$$
B((u, \phi, \theta, w),(u, \phi, \theta, w)) \geq M_{0}\|(u, \phi, \theta, w)\|_{V}^{2} .
$$

Thus, $B$ is coercive. Hence, we use the Lax-Milgram theorem to conclude that (52) has a unique solution:

$$
\begin{gathered}
u, \phi \in H_{0}^{1}(0,1), \\
w \in H_{*}^{1}(0,1), \\
\theta \in L^{2}(0,1),
\end{gathered}
$$


Substituting $u, \phi, \theta$, and $w$ into $(46)_{1,3}$, we have

$$
\nu, \varphi \in H_{0}^{1}(0,1)
$$

Similarly, the compensation of $v$ in (47) with $(46)_{7}$, gives

$$
\mathscr{Y}, \mathscr{Y}_{\rho} \in L^{2}\left((0,1) \times(0,1) \times\left(\tau_{1}, \tau_{2}\right)\right)
$$

Moreover, if we take $\widehat{u}=\widehat{\theta}=\widehat{w}=0$ in (54), we get

$$
\begin{gathered}
a_{3} \int_{0}^{1} \phi_{x} \widehat{\phi}_{x} d x+\mu_{3} \int_{0}^{1} \phi \widehat{\phi} d x+a_{2} \int_{0}^{1} u_{x} \widehat{\phi}_{x} d x \\
\quad+d \int_{0}^{1} w_{x} \widehat{\phi} d x-m \int_{0}^{1} \theta \widehat{\phi} d x \\
=\int_{0}^{1} h_{2} \widehat{\phi} d x, \quad \forall \widehat{\phi} \in H_{0}^{1}(0,1),
\end{gathered}
$$

which implies

$$
\begin{aligned}
a_{3} \int_{0}^{1} \phi_{x} \widehat{\phi}_{x} d x= & \int_{0}^{1}\left(h_{2}-\mu_{3} \phi+a_{2} u_{x x}-d w_{x}\right. \\
& +m \theta) \widehat{\phi} d x, \quad \forall \widehat{\phi} \in H_{0}^{1}(0,1),
\end{aligned}
$$

that is

$$
a_{3} \phi_{x x}=\mu_{3} \phi-a_{2} u_{x x}+d w_{x}-m \theta-h_{2} \in L^{2}(0,1) .
$$

Consequently

$$
\phi \in H^{2}(0,1) \cap H_{0}^{1}(0,1) .
$$

Similarly, we get

$$
\begin{aligned}
& u \in H^{2}(0,1) \cap H_{0}^{1}(0,1), \\
& \theta \in H_{0}^{1}(0,1),
\end{aligned}
$$

and, if we let $\widehat{u}=\widehat{\theta}=\widehat{\phi}=0$ in (54), we get

$$
\begin{aligned}
& \frac{\alpha \lambda+k_{3}}{\lambda} \int_{0}^{1} w \widehat{w} d x+\frac{k_{2}}{\lambda} \int_{0}^{1} w_{x} \widehat{w}_{x} d x+\frac{k_{1}}{\lambda} \int_{0}^{1} \theta_{x} \widehat{w} d x \\
& \quad+d \int_{0}^{1} \phi_{x} \widehat{w} d x-m \int_{0}^{1} \theta \widehat{\phi} d x \\
& =\int_{0}^{1} h_{4} \widehat{w} d x, \quad \forall \widehat{w} \in H_{*}^{1}(0,1),
\end{aligned}
$$

which implies

$$
\begin{aligned}
\frac{k_{2}}{\lambda} \int_{0}^{1} w_{x} \psi_{x} d x= & \int_{0}^{1}\left(-h_{4}+\frac{\alpha \lambda+k_{3}}{\lambda} w+\frac{k_{1}}{\lambda} \theta_{x}+d \phi_{x}\right) \psi d x, \\
& \forall \psi \in C^{1}(0,1) \subset H_{*}^{1}(0,1)
\end{aligned}
$$

Thus, using integration by parts, we get

$$
w_{x}(1) \psi(1)-w_{x}(0) \psi(0)=0, \quad \forall \psi \in C^{1}(0,1)
$$

Therefore,

$$
w_{x}(1)=w_{x}(0)=0
$$

Consequently,

$$
w \in H_{*}^{2}(0,1) \cap H_{*}^{1}(0,1)
$$

Finally, the application of regularity theory for the linear elliptic equations guarantees the existence of unique $U \in$ $\mathscr{D}(\mathscr{A})$ such that $(45)$ is satisfied.

Consequently, we conclude that $\mathscr{A}$ is a maximal dissipative operator. Hence, by Lumer-Philips theorem (see [29]), we have the well-posedness result. This completes the proof.

\section{Exponential Decay}

In this section, we prove our stability result of the system (23)-(26).

For this, we have the following lemmas.

Lemma 3. The energy functional E, defined by

$$
\begin{aligned}
E(t)= & \frac{1}{2} \int_{0}^{1}\left[\rho_{u} u_{t}^{2}+a_{1} u_{x}^{2}+\rho_{\phi} \phi_{t}^{2}+a_{3} \phi_{x}^{2}+2 a_{2} u_{x} \phi_{x}+c_{0} \theta^{2}+\alpha w^{2}\right] d x \\
& +\frac{1}{2} \int_{0}^{1} \int_{0}^{1} \int_{\tau_{1}}^{\tau_{2}} \sigma\left|\mu_{2}(\sigma)\right| \mathcal{Y}^{2}(x, \rho, \sigma, t) d \sigma d \rho d x,
\end{aligned}
$$

satisfies

$$
E^{\prime}(t) \leq-k_{2} \int_{0}^{1} w_{x}^{2} d x-k_{3} \int_{0}^{1} w^{2} d x-\eta_{0} \int_{0}^{1} \phi_{t}^{2} d x \leq 0
$$

where $\eta_{0}=\mu_{1}-\int_{\tau_{1}}^{\tau_{2}}\left|\mu_{2}(\sigma)\right| d \sigma>0$.

Proof. Multiplying Equation $(23)_{1,2,3,4}$ by $u_{t}, \phi_{t}, \theta$, and $w$, integrating by parts over $(0,1)$, and using $(26)$, we get

$$
\begin{aligned}
& \frac{1}{2} \frac{d}{d t} \int_{0}^{1}\left[\rho_{u} u_{t}^{2}+a_{1} u_{x}^{2}+\rho_{\phi} \phi_{t}^{2}+a_{3} \phi_{x}^{2}+2 a_{2} u_{x} \phi_{x}+c_{0} \theta^{2}+\alpha w^{2}\right] d x \\
& \quad+\mu_{1} \int_{0}^{1} \phi_{t}^{2} d x+\int_{0}^{1} \phi_{t} \int_{\tau_{1}}^{\tau_{2}}\left|\mu_{2}(\sigma)\right| \mathcal{Y}(x, 1, \sigma, t) d \sigma d x \\
& \quad+k_{3} \int_{0}^{1} w^{2} d x+k_{2} \int_{0}^{1} w_{x}^{2} d x=0 .
\end{aligned}
$$

Now, multiplying Equation $(23)_{5}$ by $\mathcal{Y}\left|\mu_{2}(\sigma)\right|$, and 
integrating the result over $(0,1) \times(0,1) \times\left(\tau_{1}, \tau_{2}\right)$

$$
\begin{aligned}
\frac{d}{d t} & \frac{1}{2} \int_{0}^{1} \int_{0}^{1} \int_{\tau_{1}}^{\tau_{2}} \sigma\left|\mu_{2}(\sigma)\right| \mathscr{Y}^{2}(x, \rho, \sigma, t) d \sigma d \rho d x \\
= & -\int_{0}^{1} \int_{0}^{1} \int_{\tau_{1}}^{\tau_{2}}\left|\mu_{2}(\sigma)\right| \mathscr{Y}_{\rho}(x, \rho, \sigma, t) d \sigma d \rho d x \\
= & -\frac{1}{2} \int_{0}^{1} \int_{0}^{1} \int_{\tau_{1}}^{\tau_{2}}\left|\mu_{2}(\sigma)\right| \frac{d}{d \rho} \mathscr{Y}^{2}(x, \rho, \sigma, t) d \sigma d \rho d x \\
= & \frac{1}{2} \int_{0}^{1} \int_{\tau_{1}}^{\tau_{2}}\left|\mu_{2}(\sigma)\right|\left(\mathscr{Y}^{2}(x, 0, \sigma, t)-\mathscr{Y}^{2}(x, 1, \sigma, t)\right) d \sigma d x \\
= & \frac{1}{2} \int_{\tau_{1}}^{\tau_{2}}\left|\mu_{2}(\sigma)\right| d \sigma \int_{0}^{1} \phi_{t}^{2} d x \\
& -\frac{1}{2} \int_{0}^{1} \int_{\tau_{1}}^{\tau_{2}}\left|\mu_{2}(\sigma)\right| \mathscr{Y}^{2}(x, 1, \sigma, t) d \sigma d x .
\end{aligned}
$$

Now, by substituting (78) into (77), and using Young's inequality, we have

$E^{\prime}(t) \leq-k_{3} \int_{0}^{1} w^{2} d x-k_{2} \int_{0}^{1} w_{x}^{2} d x-\left(\mu_{1}-\int_{\tau_{1}}^{\tau_{2}}\left|\mu_{2}(\sigma)\right| d \sigma\right) \int_{0}^{1} \phi_{t}^{2} d x$

then, by (27), $\exists \eta_{0}>0$ so that

$$
E^{\prime}(t) \leq-k_{3} \int_{0}^{1} w^{2} d x-k_{2} \int_{0}^{1} w_{x}^{2} d x-\eta_{0} \int_{0}^{1} \phi_{t}^{2} d x
$$

then we obtain (76) ( $E$ is a nonincreasing function).

Remark 4. Using (14), we conclude that $E(t)$ satisfies

$$
\begin{aligned}
E(t)> & \frac{1}{2} \int_{0}^{1}\left[\rho_{u} u_{t}^{2}+a_{4} u_{x}^{2}+\rho_{\phi} \phi_{t}^{2}+a_{5} \phi_{x}^{2}+c_{0} \theta^{2}+\alpha w^{2}\right] d x \\
& +\frac{1}{2} \int_{0}^{1} \int_{0}^{1} \int_{\tau_{1}}^{\tau_{2}} \sigma\left|\mu_{2}(\sigma)\right| \mathscr{Y}^{2}(x, \rho, \sigma, t) d \sigma d \rho d x,
\end{aligned}
$$

where

$$
\begin{aligned}
& a_{4}=\frac{1}{2}\left(a_{1}-\frac{a_{2}^{2}}{a_{3}}\right)>0, \\
& a_{5}=\frac{1}{2}\left(a_{3}-\frac{a_{2}^{2}}{a_{1}}\right)>0 .
\end{aligned}
$$

Then, the function $E(t)$ is nonnegative.

\section{Lemma 5. The functional}

$$
D_{1}(t):=\rho_{\phi} \int_{0}^{1} \phi_{t} \phi d x-\frac{a_{2}}{a_{1}} \rho_{u} \int_{0}^{1} \phi u_{t} d x+\frac{\mu_{1}}{2} \int_{0}^{1} \phi^{2} d x,
$$

satisfies, for any $\varepsilon_{1}>0$

$$
\begin{aligned}
D_{1}^{\prime}(t) \leq & -\frac{a}{2} \int_{0}^{1} \phi_{x}^{2} d x+\varepsilon_{1} \int_{0}^{1} u_{t}^{2} d x+c\left(1+\frac{1}{\varepsilon_{1}}\right) \int_{0}^{1} \phi_{t}^{2} d x \\
& +c \int_{0}^{1} w^{2} d x+c \int_{0}^{1} \theta^{2} d x+c \int_{0}^{1} \int_{\tau_{1}}^{\tau_{2}}\left|\mu_{2}(\sigma)\right| \mathscr{Y}^{2}(x, 1, \sigma, t) d \sigma d x
\end{aligned}
$$

Proof. Direct computation using integration by parts and Young's inequality yields

$$
\begin{aligned}
D_{1}^{\prime}(t)= & -a_{3} \int_{0}^{1} \phi_{x}^{2} d x+\rho_{\phi} \int_{0}^{1} \phi_{t}^{2} d x+\frac{a_{2}^{2}}{a_{1}} \int_{0}^{1} \phi_{x}^{2} d x \\
& +\frac{a_{2}}{a_{1}} \rho_{u} \int_{0}^{1} \phi_{t} u_{t} d x-d \int_{0}^{1} w_{x} \phi d x+m \int_{0}^{1} \theta \phi d x \\
& +\frac{a_{2} \gamma}{a_{1}} \int_{0}^{1} \theta_{x} \phi d x-\int_{0}^{1} \phi \int_{\tau_{1}}^{\tau_{2}}\left|\mu_{2}(\sigma)\right| \mathcal{Y}(x, 1, \sigma, t) d \sigma d x \\
& \leq-\left(a_{3}-\frac{a_{2}^{2}}{a_{1}}\right) \int_{0}^{1} \phi_{x}^{2} d x+\rho_{\phi} \int_{0}^{1} \phi_{t}^{2} d x \\
& +\frac{a_{2}}{a_{1}} \rho_{u} \int_{0}^{1} \phi_{t} u_{t} d x-d \int_{0}^{1} w_{x} \phi d x+m \int_{0}^{1} \theta \phi d x \\
& +\frac{a_{2} \gamma}{a_{1}} \int_{0}^{1} \theta_{x} \phi d x-\int_{0}^{1} \phi \int_{\tau_{1}}^{\tau_{2}}\left|\mu_{2}(\sigma)\right| \mathcal{Y}(x, 1, \sigma, t) d \sigma d x
\end{aligned}
$$

We use Cauchy-Schwartz, Young's, and Poincare's inequalities; for $\delta_{1}, \varepsilon_{1}>0$, we obtain

$$
\begin{aligned}
D_{1}^{\prime}(t) \leq & -\left(a_{3}-\frac{a_{2}^{2}}{a_{1}}-\mu_{1} c \delta_{1}\right) \int_{0}^{1} \phi_{x}^{2} d x+\varepsilon_{1} \int_{0}^{1} u_{t}^{2} d x \\
& +c\left(1+\frac{1}{\varepsilon_{1}}\right) \int_{0}^{1} \phi_{t}^{2} d x+d \int_{0}^{1} w \phi_{x} d x+m \int_{0}^{1} \theta \phi d x \\
& -\frac{a_{2} \gamma}{a_{1}} \int_{0}^{1} \theta \phi_{x} d x+\frac{1}{4 \delta_{1}} \int_{0}^{t} \int_{\tau_{1}}^{\tau_{2}}\left|\mu_{2}(\sigma)\right| \mathscr{Y}^{2}(x, 1, \sigma, t) d \sigma d x
\end{aligned}
$$

Bearing in mind (14), and letting $\delta_{1}=a / 2 c$, we obtain the estimate (84).

Lemma 6. The functional

$$
D_{2}(t):=a_{2}\left(\int_{0}^{1} \phi_{t} u d x-\int_{0}^{1} \phi u_{t} d x\right)
$$

satisfies,

$$
\begin{aligned}
D_{2}^{\prime}(t) \leq & -\frac{a_{2}^{2}}{2 \rho_{\phi}} \int_{0}^{1} u_{x}^{2} d x+c \int_{0}^{1} \phi_{x}^{2} d x+c \int_{0}^{1} \phi_{t}^{2} d x+c \int_{0}^{1} w^{2} d x \\
& +c \int_{0}^{1} \theta^{2} d x+c \int_{0}^{1} \int_{\tau_{1}}^{\tau_{2}}\left|\mu_{2}(\sigma)\right| \mathcal{Y}^{2}(x, 1, \sigma, t) d \sigma d x .
\end{aligned}
$$


Proof. By differentiating $D_{2}$, then using (23), integration by parts, and (26), we obtain

$$
\begin{aligned}
D_{2}^{\prime}(t)= & -\frac{a_{2}^{2}}{\rho_{\phi}} \int_{0}^{1} u_{x}^{2} d x+\frac{a_{2}^{2}}{\rho_{u}} \int_{0}^{1} \phi_{x}^{2} d x-\left(\frac{a_{2} a_{3}}{\rho_{\phi}}-\frac{a_{1} a_{2}}{\rho_{u}}\right) \int_{0}^{1} \phi_{x} u_{x} d x \\
& -\frac{a_{2} \mu_{1}}{\rho_{\phi}} \int_{0}^{1} u \phi_{t} d x+\frac{a_{2} d}{\rho_{\phi}} \int_{0}^{1} w u_{x} d x+\frac{a_{2} m}{\rho_{\phi}} \int_{0}^{1} \theta u d x \\
& -\frac{a_{2} \gamma}{\rho_{u}} \int_{0}^{1} \theta \phi_{x} d x-\frac{a_{2}}{\rho_{\phi}} \int_{0}^{1} u \int_{\tau_{1}}^{\tau_{2}}\left|\mu_{2}(\sigma)\right| \mathcal{Y}(x, 1, \sigma, t) d \sigma d x
\end{aligned}
$$

Now, we estimate the last six terms in the RHS of (89), using Young's, Cauchy-Schwartz, and Poincare's inequalities. For $\delta_{2}, \delta_{3}, \delta_{4}, \delta_{5}, \delta_{6}>0$, we have

$$
\begin{gathered}
-\left(\frac{a_{2} a_{3}}{\rho_{\phi}}-\frac{a_{1} a_{2}}{\rho_{u}}\right) \int_{0}^{1} \phi_{x} u_{x} d x \leq \delta_{2} \int_{0}^{1} u_{x}^{2} d x+\left(\frac{a_{2} a_{3}}{\rho_{\phi}}-\frac{a_{1} a_{2}}{\rho_{u}}\right)^{2} \frac{1}{4 \delta_{2}} \int_{0}^{1} \phi^{2} d x . \\
\frac{a_{2} d}{\rho_{\phi}} \int_{0}^{1} w u_{x} d x \leq \delta_{3} \int_{0}^{1} u_{x}^{2} d x+\frac{c}{4 \delta_{3}} \int_{0}^{1} w^{2} d x, \\
-\frac{a_{2} \mu_{1}}{\rho_{\phi}} \int_{0}^{1} u \phi_{t} d x \leq c \delta_{4} \int_{0}^{1} u_{x}^{2} d x+\frac{c}{4 \delta_{4}} \int_{0}^{1} \phi_{t}^{2} d x,
\end{gathered}
$$

and

$$
\begin{gathered}
\frac{a_{2} m}{\rho_{\phi}} \int_{0}^{1} \theta u d x \leq \delta_{5} c \int_{0}^{1} u_{x}^{2} d x+\frac{c}{4 \delta_{5}} \int_{0}^{1} \theta^{2} d x \\
-\frac{a_{2}}{\rho_{\phi}} \int_{0}^{1} u \int_{\tau_{1}}^{\tau_{2}}\left|\mu_{2}(\sigma)\right| \mathcal{Y}(x, 1, \sigma, t) d \sigma d x \leq c \delta_{6} \int_{0}^{1} u_{x}^{2} d x \\
-\frac{c}{4 \delta_{6}} \int_{0}^{1} \int_{\tau_{1}}^{\tau_{2}}\left|\mu_{2}(\sigma)\right| \mathscr{Y}^{2}(x, 1, \sigma, t) d \sigma d x .
\end{gathered}
$$

By letting $\delta_{2}=\delta_{3}=a_{2} / 10 \rho_{\phi}, \delta_{4}=\delta_{5}=\delta_{6}=a_{2} / 10 c \rho_{\phi}$, and substituting into (89), we get (88).

\section{Lemma 7. The functional}

$$
D_{3}(t):=-\rho_{u} \int_{0}^{1} u_{t} u d x
$$

satisfies

$D_{3}^{\prime}(t) \leq-\rho_{u} \int_{0}^{1} u_{t}^{2} d x+3 a_{1} \int_{0}^{1} u_{x}^{2} d x+\frac{a_{3}}{4} \int_{0}^{1} \phi_{x}^{2} d x+\frac{\gamma^{2}}{4 a_{1}} \int_{0}^{1} \theta^{2} d x$.
Proof. Direct computations give

$$
D_{3}^{\prime}(t)=-\rho_{u} \int_{0}^{1} u_{t}^{2} d x+a_{1} \int_{0}^{1} u_{x}^{2} d x+a_{2} \int_{0}^{1} u_{x} \phi_{x} d x-\gamma \int_{0}^{1} u_{x} \theta d x
$$

Estimating (93) easily follows by using Young's inequality and (14).

Lemma 8. The functional

$$
D_{4}(t):=-c_{0} \alpha \int_{0}^{1} \theta\left(\int_{0}^{x} w(y) d y\right) d x
$$

satisfies

$$
\begin{aligned}
D_{4}^{\prime}(t) \leq & -\frac{c_{0} k_{1}}{2} \int_{0}^{1} \theta^{2} d x+\varepsilon_{2} \int_{0}^{1} u_{t}^{2} d x+c\left(1+\frac{1}{\varepsilon_{2}}\right) \int_{0}^{1} w^{2} d x \\
& +c \int_{0}^{1} \phi_{t}^{2} d x+c \int_{0}^{1} w_{x}^{2} d x .
\end{aligned}
$$

Proof. Direct computations give

$$
\begin{aligned}
D_{4}^{\prime}(t)= & -c_{0} k_{1} \int_{0}^{1} \theta^{2} d x+\alpha k_{1} \int_{0}^{1} w^{2} d x+\alpha \gamma \int_{0}^{1} u_{t} w d x \\
& +c_{0} d \int_{0}^{1} \theta \phi_{t} d x-\alpha m \int_{0}^{1} \phi_{t}\left(\int_{0}^{x} w(y) d y\right) d x \\
& +c_{0} k_{2} \int_{0}^{1} w_{x} \theta d x-c_{0} k_{3} \int_{0}^{1} \theta\left(\int_{0}^{x} w(y) d y\right) d x
\end{aligned}
$$

Estimate (96) easily follows by using Young's and Cauchy-Schwartz inequalities.

Now, let us introduce the following functional used by.

Lemma 9. The functional

$$
D_{5}(t):=\int_{0}^{1} \int_{0}^{1} \int_{\tau_{1}}^{\tau_{2}} \sigma e^{-\sigma \rho}\left|\mu_{2}(\sigma)\right| \mathscr{Y}^{2}(x, \rho, \sigma, t) d \sigma d \rho d x
$$

satisfies

$$
\begin{aligned}
D_{5}^{\prime}(t) \leq & -\eta_{1} \int_{0}^{1} \int_{0}^{1} \int_{\tau_{1}}^{\tau_{2}} \sigma\left|\mu_{2}(\sigma)\right| \mathscr{Y}^{2}(x, \rho, \sigma, t) d \sigma d \rho d x \\
& +\mu_{1} \int_{0}^{1} \phi_{t}^{2} d x-\eta_{1} \int_{0}^{1} \int_{\tau_{1}}^{\tau_{2}}\left|\mu_{2}(\sigma)\right| \mathcal{Y}^{2}(x, 1, \sigma, t) d \sigma d x
\end{aligned}
$$

where $\eta_{1}>0$. 
Proof. By differentiating $D_{5}$, with respect to $t$ and using the last equation in (23), we have

$$
\begin{aligned}
D_{5}^{\prime}(t)= & -2 \int_{0}^{1} \int_{0}^{1} \int_{\tau_{1}}^{\tau_{2}} e^{-\sigma \rho}\left|\mu_{2}(\sigma)\right| \mathscr{Y}_{\rho}(x, \rho, \sigma, t) d \sigma d \rho d x \\
= & -\int_{0}^{1} \int_{0}^{1} \int_{\tau_{1}}^{\tau_{2}} \sigma e^{-\sigma \rho}\left|\mu_{2}(\sigma)\right| \mathscr{Y}^{2}(x, \rho, \sigma, t) d \sigma d \rho d x \\
& -\int_{0}^{1} \int_{\tau_{1}}^{\tau_{2}}\left|\mu_{2}(\sigma)\right|\left[e^{-\sigma} \mathscr{Y}^{2}(x, 1, \sigma, t)-\mathscr{Y}^{2}(x, 0, \sigma, t)\right] d \sigma d x .
\end{aligned}
$$

Using the fact that $\mathscr{Y}(x, 0, \sigma, t)=\phi_{t}(x, t)$, and $e^{-\sigma} \leq e^{-\sigma \rho}$ $\leq 1$, for all $0<\rho<1$, we obtain

$$
\begin{aligned}
D_{5}^{\prime}(t)= & -\eta_{1} \int_{0}^{1} \int_{0}^{1} \int_{\tau_{1}}^{\tau_{2}} \sigma\left|\mu_{2}(\sigma)\right| \mathscr{Y}^{2}(x, \rho, \sigma, t) d \sigma d \rho d x \\
& -\int_{0}^{1} \int_{\tau_{1}}^{\tau_{2}} e^{-\sigma}\left|\mu_{2}(\sigma)\right| \mathscr{Y}^{2}(x, 1, \sigma, t) d \sigma d x \\
& +\left(\int_{\tau_{1}}^{\tau_{2}}\left|\mu_{2}(\sigma)\right| d \sigma\right) \int_{0}^{1} \phi_{t}^{2} d x
\end{aligned}
$$

Because $-e^{-\sigma}$ is a increasing function, we have $-e^{-\sigma}$ $\leq-e^{-\tau_{2}}$, for all $\sigma \in\left[\tau_{1}, \tau_{2}\right]$.

Finally, setting $\eta_{1}=e^{-\tau_{2}}$ and recalling (27), we find (99). We are now ready to prove the main result.

Theorem 10. Assume (27) holds. Then, $\forall t_{0}>0$, there exist $\beta_{1}, \beta_{2}>0$ such that the energy functional given by (75) satisfies

$$
E(t) \leq \beta_{1} e^{-\beta_{2} t}, \quad \forall t \geq 0 .
$$

Proof. We define the functional of Lyapunov

$$
\begin{aligned}
\mathscr{L}(t):= & N E(t)+N_{1} D_{1}(t)+N_{2} D_{2}(t) \\
& +D_{3}(t)+N_{4} D_{4}(t)+N_{5} D_{5}(t),
\end{aligned}
$$

where $N, N_{1}, N_{2}, N_{4}, N_{5}>0$ we will assign them later.

By differentiating (103) and using (75), (84), (88), (93), (96), and (99), we have

$$
\begin{aligned}
\mathscr{L}^{\prime}(t) \leq & -\left[\frac{a N_{1}}{2}-c N_{2}-\frac{a_{3}}{4}\right] \int_{0}^{1} \phi_{x}^{2} d x-\left[\rho_{u}-N_{1} \varepsilon_{1}-N_{4} \varepsilon_{2}\right] \int_{0}^{1} u_{t}^{2} d x \\
& -\left[\frac{a_{2}^{2} N_{2}}{2 \rho_{\phi}}-3 a_{1}\right] \int_{0}^{1} u_{x}^{2} d x-\left[k_{2} N-c N_{4}\right] \int_{0}^{1} w_{x}^{2} d x \\
& -\left[\eta_{0} N-c N_{1}\left(1+\frac{1}{\varepsilon_{1}}\right)-N_{2} c-N_{4} c-\mu_{1} N_{5}\right] \int_{0}^{1} \phi_{t}^{2} d x \\
& -\left[k_{3} N-c N_{1}-c N_{2}-c N_{4}\left(1+\frac{1}{\varepsilon_{2}}\right)\right] \int_{0}^{1} w^{2} d x
\end{aligned}
$$

$$
\begin{aligned}
& -\left[\frac{c_{0} k_{1} N_{4}}{2}-c N_{1}-c N_{2}-\frac{\gamma^{2}}{4 a_{1}}\right] \int_{0}^{1} \theta^{2} d x \\
& -\left[N_{5} \eta_{1}-c N_{1}-c N_{2}\right] \int_{0}^{1} \int_{\tau_{1}}^{\tau_{2}}\left|\mu_{2}(\sigma)\right| \mathscr{Y}^{2}(x, 1, \sigma, t) d \sigma d x \\
& -N_{5} \eta_{1} \int_{0}^{1} \int_{0}^{1} \int_{\tau_{1}}^{\tau_{2}} \sigma\left|\mu_{2}(\sigma)\right| \mathscr{Y}^{2}(x, \rho, \sigma, t) d \sigma d \rho d x .
\end{aligned}
$$

By setting

$$
\varepsilon_{1}=\frac{\rho_{u}}{4 N_{1}}, \quad \varepsilon_{2}=\frac{\rho_{u}}{4 N_{4}},
$$

we obtain

$$
\begin{aligned}
\mathscr{L}^{\prime}(t) \leq & -\left[\frac{a N_{1}}{2}-c N_{2}-\frac{a_{3}}{4}\right] \int_{0}^{1} \phi_{x}^{2} d x-\left[\frac{\rho_{u}}{2}\right] \int_{0}^{1} u_{t}^{2} d x \\
& -\left[\frac{a_{2}^{2} N_{2}}{2 \rho_{\phi}}-3 a_{1}\right] \int_{0}^{1} u_{x}^{2} d x-\left[k_{2} N-c N_{4}\right] \int_{0}^{1} w_{x}^{2} d x \\
& -\left[\eta_{0} N-c N_{1}\left(1+N_{1}\right)-N_{2} c-N_{4} c-\mu_{1} N_{5}\right] \int_{0}^{1} \phi_{t}^{2} d x \\
& -\left[k_{3} N-c N_{1}-c N_{2}-c N_{4}\left(1+N_{4}\right)\right] \int_{0}^{1} w^{2} d x \\
& -\left[\frac{c_{0} k_{1} N_{4}}{2}-c N_{1}-c N_{2}-\frac{\gamma^{2}}{4 a_{1}}\right] \int_{0}^{1} \theta^{2} d x \\
& -\left[N_{5} \eta_{1}-c N_{1}-c N_{2}\right] \int_{0}^{1} \int_{\tau_{1}}^{\tau_{2}}\left|\mu_{2}(\sigma)\right| \mathscr{Y}^{2}(x, 1, \sigma, t) d \sigma d x \\
& -N_{5} \eta_{1} \int_{0}^{1} \int_{0}^{1} \int_{\tau_{1}}^{\tau_{2}} \sigma\left|\mu_{2}(\sigma)\right| \mathscr{Y}^{2}(x, \rho, \sigma, t) d \sigma d \rho d x .
\end{aligned}
$$

At this point, we choose our constants.

We choose $\mathrm{N}_{2}$ large enough so that

$$
\alpha_{1}=\frac{a_{2}^{2} N_{2}}{2 \rho_{\phi}}-3 a_{1}>0
$$

then we pick $N_{1}$ large enough such that

$$
\alpha_{2}=\frac{a N_{1}}{2}-c N_{2}-\frac{a_{3}}{4}>0,
$$

then we select $N_{4}$ and $N_{5}$ large enough such that

$$
\begin{aligned}
& \alpha_{3}=\frac{c_{0} k_{1} N_{4}}{2}-c N_{1}-c N_{2}-\frac{\gamma^{2}}{4 a_{1}}>0, \\
& \alpha_{4}=N_{5} \eta_{1}-c N_{1}-c N_{2}>0 .
\end{aligned}
$$


Thus, we arrive at

$$
\begin{aligned}
\mathscr{L}^{\prime}(t) \leq & -\alpha_{2} \int_{0}^{1} \phi_{x}^{2} d x-\frac{\rho_{u}}{2} \int_{0}^{1} u_{t}^{2} d x-\alpha_{1} \int_{0}^{1} u_{x}^{2} d x \\
& -\left[\eta_{0} N-c\right] \int_{0}^{1} \phi_{t}^{2} d x-\left[k_{3} N-c\right] \int_{0}^{1} w^{2} d x \\
& -\left[k_{2} N-c\right] \int_{0}^{1} w_{x}^{2} d x-\alpha_{3} \int_{0}^{1} \theta^{2} d x \\
& -\alpha_{4} \int_{0}^{1} \int_{\tau_{1}}^{\tau_{2}}\left|\mu_{2}(\sigma)\right| \mathscr{Y}^{2}(x, 1, \sigma, t) d \sigma d x \\
& -\alpha_{5} \int_{0}^{1} \int_{0}^{1} \int_{\tau_{1}}^{\tau_{2}} \sigma\left|\mu_{2}(\sigma)\right| \mathscr{Y}^{2}(x, \rho, \sigma, t) d \sigma d \rho d x .
\end{aligned}
$$

where $\alpha_{5}=\eta_{1} N_{5}$.

On the other hand, if we let

$$
\mathfrak{L}(t)=N_{1} D_{1}(t)+N_{2} D_{2}(t)+D_{3}(t)+N_{4} D_{4}(t)+N_{5} D_{5}(t),
$$

then

$$
\begin{aligned}
|\mathfrak{Q}(t)| \leq & N_{1} \rho_{\phi} \int_{0}^{1}\left|\phi \phi_{t}\right| d x+N_{1} \frac{a_{2}}{a_{1}} \rho_{u} \int_{0}^{1}\left|\phi u_{t}\right| d x+N_{1} \frac{\mu_{1}}{2} \int_{0}^{1} \phi^{2} d x \\
& +N_{2} a_{2} \int_{0}^{1}\left|\phi u_{t}-u \phi_{t}\right| d x+\rho_{u} \int_{0}^{1}\left|u_{t} u\right| d x \\
& +N_{4} c_{0} \alpha \int_{0}^{1}\left|\theta\left(\int_{0}^{x} w(y) d y\right)\right| d x \\
& +N_{5} \int_{0}^{1} \int_{0}^{1} \int_{\tau_{1}}^{\tau_{2}} \sigma e^{-\sigma \rho}\left|\mu_{2}(\sigma)\right| \mathscr{Y}^{2}(x, \rho, \sigma, t) d \sigma d \rho d x .
\end{aligned}
$$

According Young's, Cauchy-Schwartz, and Poincaré inequalities, we find

$$
\begin{aligned}
|\mathfrak{Q}(t)| \leq & c \int_{0}^{1}\left(u_{t}^{2}+\phi_{t}^{2}+\phi_{x}^{2}+u_{x}^{2}+\theta^{2}+w^{2}\right) d x \\
& +c \int_{0}^{1} \int_{0}^{1} \int_{\tau_{1}}^{\tau_{2}} \sigma\left|\mu_{2}(\sigma)\right| \mathscr{Y}^{2}(x, \rho, \sigma, t) d \sigma d \rho .
\end{aligned}
$$

On the other hand, we can write

$$
\begin{aligned}
a_{1} u_{x}^{2}+2 a_{2} \phi_{x} u_{x}+a_{4} \phi_{x}^{2}= & \frac{1}{2}\left[a_{1}\left(u_{x}+\frac{a_{2}}{a_{1}} \phi_{x}\right)^{2}+a_{4}\left(\phi_{x}+\frac{a_{2}}{a_{4}} u_{x}\right)^{2}\right. \\
& \left.+\left(a_{1}-\frac{a_{2}^{2}}{a_{4}}\right) u_{x}^{2}+\left(a_{4}-\frac{a_{2}^{2}}{a_{1}}\right) \phi_{x}^{2}\right] .
\end{aligned}
$$

Since $a_{1} a_{3}>a_{2}^{2}$ and (27), we deduce that

$$
a_{1} u_{x}^{2}+2 a_{2} \phi_{x} u_{x}+a_{4} \phi_{x}^{2}>\frac{1}{2}\left[\left(a_{1}-\frac{a_{2}^{2}}{a_{4}}\right) u_{x}^{2}+\left(a_{4}-\frac{a_{2}^{2}}{a_{1}}\right) \phi_{x}^{2}\right] .
$$

Hence, we get

$$
|\mathfrak{Q}(t)|=|\mathscr{L}(t)-N E(t)| \leq c E(t),
$$

that is,

$$
(N-c) E(t) \leq \mathscr{L}(t) \leq(N+c) E(t) .
$$

At this point, we choose $N$ large enough such that

$$
N-c>0, N \eta_{0}-c>0, N k_{3}-c>0, N k_{2}-c>0,
$$

and exploiting (75), the estimates (110) and (117), respectively, gives

$$
c_{2} E(t) \leq \mathscr{L}(t) \leq c_{3} E(\mathrm{t}), \quad \forall t \geq 0,
$$

$$
\mathscr{L}^{\prime}(t) \leq-d_{1} E(t), \quad \forall t \geq 0,
$$

for some $d_{1}, c_{2}, c_{3}>0$.

Consequently, for some $\beta_{2}>0$, we find

$$
\mathscr{L}^{\prime}(t) \leq-\beta_{2} \mathscr{L}(t), \quad \forall t \geq 0 .
$$

Integration of $(120)$ over $(0, t)$ gives

$$
\mathscr{L}(t) \leq \mathscr{L}(0) e^{-\beta_{2} t}, \quad \forall t \geq 0 .
$$
(121)

Consequently, (102) is established by virtue of (117) and

\section{Data Availability}

No data were used to support the study.

\section{Conflicts of Interest}

This work does not have any conflicts of interest.

\section{Acknowledgments}

The sixth author extend their appreciation to the Deanship of Scientific Research at King Khalid University for funding this work through research group program under grant (R.G.P.1/3/42).

\section{References}

[1] A. C. Eringen, "A continuum theory of swelling porous elastic soils," International Journal of Engineering Science, vol. 32, no. 8, pp. 1337-1349, 1994. 
[2] A. Bedford and D. S. Drumheller, "Theories of immiscible and structured mixtures," International Journal of Engineering Science, vol. 21, no. 8, pp. 863-960, 1983.

[3] J. E. Bowels, Foundation Design and Analysis, McGraw Hill Inc., New York, U.S.A, 1988.

[4] V. Q. Hung, Hidden Disaster, University of Saska Techwan, Saskatoon, Canada, University News, 2003.

[5] D. Iesan, "On the theory of mixtures of thermoelastic solids," Journal of Thermal Stresses, vol. 14, no. 4, pp. 389-408, 1991.

[6] L. D. Jones and I. Jefferson, Expansive Soils, ICE Publishing, London, 2012.

[7] B. Kalantari, "Engineering significant of swelling soils," Research Journal of Applied Sciences, Engineering and Technology, vol. 4, no. 17, pp. 2874-2878, 2012.

[8] R. Quintanilla, "Exponential stability for one-dimensional problem of swelling porous elastic soils with fluid saturation," Journal of Computational and Applied Mathematics, vol. 145, no. 2, pp. 525-533, 2002.

[9] J. M. Wang and B. Z. Guo, "On the stability of swelling porous elastic soils with fluid saturation by one internal damping," IMA Journal of Applied Mathematics, vol. 71, no. 4, pp. 565582, 2006.

[10] T. A. Apalara, "General stability result of swelling porous elastic soils with a viscoelastic damping," Zeitschrift für Angewandte Mathematik und Physik, vol. 71, no. 6, p. 200, 2020.

[11] A. Menaceur, S. Boulaaras, A. Makhlouf, K. Rajagobal, and M. Abdalla, "Limit cycles of a class of perturbed differential systems via the first-order averaging method," Complexity, vol. 2021, Article ID 5581423, 6 pages, 2021.

[12] R. Quintanilla, "Existence and exponential decay in the linear theory of viscoelastic mixtures," European Journal of Mechanics - A/Solids, vol. 24, no. 2, pp. 311-324, 2005.

[13] R. Quintanilla, "Exponential stability of solutions of swelling porous elastic soils," Meccanica, vol. 39, no. 2, pp. 139-145, 2004.

[14] R. Quintanilla, "On the linear problem of swelling porous elastic soils with incompressible fluid," International Journal of Engineering Science, vol. 40, no. 13, pp. 1485-1494, 2002.

[15] D. Ouchenane, A. Choucha, M. Abdalla, S. Boulaaras, and B. Cherif, "On the porous-elastic system with thermoelasticity of type III and distributed delay: well-posedness and stability," Journal of Function Spaces, vol. 2021, Article ID 9948143, 12 pages, 2021.

[16] T. A. Apalara, "General decay of solution in one-dimensional porous-elastic system with memory," Journal of Mathematical Analysis and Applications, vol. 469, no. 2, pp. 457-471, 2017.

[17] T. A. Apalara, "On the stabilization of a memory-type porous thermoelastic system," Bulletin of the Malaysian Mathematical Sciences Society, vol. 43, no. 2, pp. 1433-1448, 2020.

[18] A. Choucha, D. Ouchenane, and K. Zennir, "General decay of solutions in one-dimensional porous-elastic with memory and distributed delay term," Tamkang Journal of Mathematics, vol. 52, 2021.

[19] A. Choucha, D. Ouchenane, and S. Boulaaras, "Well posedness and stability result for a thermoelastic laminated Timoshenko beam with distributed delay term," Mathematical Methods in the Applied Sciences, vol. 43, no. 17, pp. 9983-10004, 2020.

[20] A. Choucha, D. Ouchenane, S. Boulaaras, B. Cherif, and M. Abdalla, "Well-posedness and stability result of the nonlinear thermodiffusion full von Kármán beam with thermal effect and time-varying delay," Journal of Function Spaces, vol. 2021, Article ID 9974034, 16 pages, 2021.

[21] A. Choucha, S. M. Boulaaras, D. Ouchenane, B. B. Cherif, and M. Abdalla, "Exponential stability of swelling porous elastic with a viscoelastic damping and distributed delay term," Journal of Function Spaces, vol. 2021, Article ID 5581634, 8 pages, 2021.

[22] A. Choucha, S. Boulaaras, D. Ouchenane, S. Alkhalaf, I. Mekawy, and M. Abdalla, "On the system of coupled nondegenerate Kirchhoff equations with distributed delay: global existence and exponential decay," Journal of Function Spaces, vol. 2021, Article ID 5577277, 13 pages, 2021.

[23] M. J. Dos Santos, B. Feng, D. S. Almeida Junior, and M. L. Santos, "Global and exponential attractors for a nonlinear porous elastic system with delay term," Discrete \& Continuous Dynamical Systems - B, vol. 26, no. 5, pp. 2805-2828, 2021.

[24] A. S. Nicaise and C. Pignotti, "Stabilization of the wave equation with boundary or internal distributed delay," Differential and Integral Equations, vol. 21, no. 9-10, pp. 935-958, 2008.

[25] H. Dridi and A. Djebabla, "On the stabilization of linear porous elastic materials by microtemperature effect and porous damping," Annali Dell Universita di Ferrara, vol. 66, pp. 13-25, 2020.

[26] D. Ieşan, "Thermoelasticity of bodies with microstructure and microtemperatures," International Journal of Solids and Structures, vol. 44, pp. 8648-8662, 2007.

[27] D. Ieşan, "On a theory of micromorphic elastic solids with microtemperatures," Journal of Thermal Stresses, vol. 24, pp. 737-752, 2001.

[28] D. IeÅŸan and R. Quintanilla, "On a theory of thermoelasticity with microtemperature," Journal of Thermal Stresses, vol. 23, pp. 199-215, 2000.

[29] A. Pazy, Semigroups of linear operateus and applications to partial differential equations, Vol 44 of applied Math. Sciences, Springer-Verlag, New York, 1983. 